\title{
NANO-VESICLES OF SALBUTAMOL SULPHATE IN METERED DOSE INHALERS: FORMULATION, CHARACTERIZATION AND IN VITRO EVALUATION
}

\author{
MONA G. ARAFA*a,b, BASSAM M AYOUBc
}

aPharmaceutics Department, Faculty of Pharmacy, The British University in Egypt, Cairo 11837, Egypt, bChemotheraputic Unit, Mansoura University Hospitals, Mansoura 35516, Egypt, cPharmaceutical Chemistry Department, Faculty of Pharmacy, The British University in

Egypt, Cairo 11837, Egypt

Email: mona.arafa@bue.edu.eg

Received: 07 Sep 2017, Revised and Accepted: 10 Oct 2017

\section{ABSTRACT}

Objective: The present work was aimed to prepare niosomes entrapping salbutamol sulphate (SS) using reversed phase evaporation method (REV).

Methods: Niosomes were prepared by mixing span 60 and cholesterol in 1:1 molar ratio in chloroform, SS in water was then added to organic phase to form niosomal SS. Formulations after evaporation of chloroform, freeze centrifuged then lyophilized, were evaluated for particles size, polydispersity index (Pdi), zeta-potential, morphology, entrapment efficiency (EE\%) and in vitro release. For pulmonary delivery; metered dose inhalers (MDI) were prepared by suspending SS niosomes equivalent to $20 \mathrm{mg}$ SS in hydrofluoroalkane (HFA). The metered valve was investigated for leakage rate, the total number of puffs/canister, weight/puff, dose uniformity and particle size.

Results: The results showed spherical niosomes with 400-451 nm particles that entrapped $66.19 \%$ of SS. 76.54 $\pm 0.132 \%$ SS release from niosomes that showed a controlled release profile for $8 \mathrm{~h}$. The leakage test was not exceeding $4 \mathrm{mg} / 3 \mathrm{~d}$, the number of puffs were up to $200 \mathrm{puffs} / \mathrm{canister}$, the dose delivered/puff was $0.1 \mathrm{mg}$ and $0.64-4.51 \mu \mathrm{m}$ niosomal aerosol.

Conclusion: The results indicate an encouraging strategy to formulate a controlled drug delivery by entrapping (SS) in niosomes which could be packaged into (MDI) that met the requirements of (USP) aerosols guidelines which offering a novel approach to respiratory delivery.

Keywords: Pulmonary drug delivery, Metered dose inhalers, Controlled release, Salbutamol sulphate, Niosomes, Mastersizer

(C) 2017 The Authors. Published by Innovare Academic Sciences Pvt Ltd. This is an open access article under the CC BY license (http://creativecommons.org/licenses/by/4.0/) DOI: http://dx.doi.org/10.22159/ijap.2017v9i6.22448

\section{INTRODUCTION}

The pulmonary delivery route is characterized with rapid onset, non-invasive nature and lack of proteolytic enzymes in lungs compared to gastrointestinal tract. In addition, onset is more rapid in case of localized delivery to lungs by inhaled medications [1-3]. Metered dose inhalers (MDIs) effectively made asthma medications simple for patients to administer to themselves. The combination of the large dispersion area of both aerosolized drug delivery systems and lungs synergistically facilitate the rapid uptake of the drug into systemic circulation offering a controlled method to deliver a known therapeutic amount with each dose [4-5]. Niosome is one of the most promising nano-carriers that can be used in targeted drug delivery systems in a controlled manner [6] showing bioavailability enhancement, continuous therapeutic effect over a longer period of time, preventing the excess drug from pouring into the systemic circulation, protecting drugs from degradation in vivo and therefore, results in reduced side-effects [7-8].

Salbutamol sulphate (SS) was developed and indicated for the symptomatic relief and prevention of bronchospasm due to bronchial asthma, chronic bronchitis and other chronic Broncho pulmonary disorders in which bronchospasm is a complicating factor. In addition, it prevents exercise-induced bronchospasm. SS is subjected to the first-pass metabolism and rapidly absorbed from the gastrointestinal tract when taken orally [9]. SS is a good candidate for controlled release formulations since its short half-life (2-4 h) that necessitates frequent administration to maintain constant therapeutic drug levels, in addition to its high water solubility [10]. Also, Anti-asthmatic drugs have high potential to induce toxic side effects after parenteral administration; stimulation of beta receptors, occur in the body. This effect results in cardiac stimulation by receptors and peripheral vasodilatation and hypotension [11]. The aforementioned facts directed our interest to design a new nano-based SS MDI according to the USP guidelines to reduce the uptake of SS by $\beta_{1}$ receptors and to deliver the drug in a controlled manner which reduces the recurrent doses and consequently reduces its toxic effect.

\section{MATERIALS AND METHODS}

\section{Experimental}

\section{Materials and reagents}

Salbutamol sulphate $(99.90 \%)$, propellant HFA and soya leicithin were kindly supplied by The Arab Drug Co., Egypt. Cholesterol (95\% stabilized) was purchased from Acros organics, (U. K.). Span 60 and chloroform were purchased from Sigma-Aldrich, Germany.

\section{Instruments}

Ultraviolet-visible spectrophotometer (V-630, Jasco, Japan), Digital precise Shaking Water Bath (WSB-18, Dahan Scientific Co. Ltd., Korea), Scanning electron microscope (JEOL 5500 LV., Tokyo, Japan), Transmission electron microscope (JEOL JSM-6510 LV., Tokyo, Japan), Rotary evaporator (OSB-2100, N-1200A, Shanghai Eyela Co. Ltd., China), Freeze centrifuge (2-16KL, Sigma Laborzentrifugen GmbH, Germany), (PAMASOL 2015, Switzerland) and Malvern Instruments Ltd (Zetasizer Nano-Zs90, MPT-Z, UK) were used.

\section{Preparation of niosomes}

Niosomes containing SS were prepared by reversed-phase evaporation method (REV) that representing formula (N). A mixture of Span 60 and cholesterol (1:1 molar ratio) was dissolved in chloroform. Subsequently, $0.5 \mathrm{~g} \mathrm{SS}$ was dissolved in water then the aqueous phase was added to the lipid phase. The mixture was then emulsified using bath sonicator for $10 \mathrm{~min}$ at $10^{\circ} \mathrm{C}$. The emulsion was rotaryevaporated at $40{ }^{\circ} \mathrm{C}$ with a rotating speed of $50 \mathrm{rpm}$ for about $15 \mathrm{~min}$ to remove the organic solvent; Traces of chloroform were eliminated by employing rotary evaporator for extra $10 \mathrm{~min}$. The suspension then was centrifuged using a refrigerated centrifuge at $4{ }^{\circ} \mathrm{C}, 10.000 \mathrm{rpm}$ for $1 \mathrm{~h}$, lyophilized and kept at $4^{\circ} \mathrm{C}$ for further investigations [12-13].

\section{Characterization of niosomes}

\section{Scanning electron microscopy (SEM) of niosomes}

Samples were sprinkled on SEM holder with double-sided adhesive tape, coated with a layer of $150 \AA$ gold for two minutes by SPI 
module TM gold sputter coater and examined using a high vacuum mode of SEM [14].

\section{Transmission electron microscopy (TEM) of niosomes}

A 10-fold aqueous diluted drop of the niosomal dispersion was subjected to collodion-coated 300 mesh copper grid, left for $5 \mathrm{~min}$, adsorbed using filter paper then a drop of $2 \%$ aqueous uranyl acetate was applied for 1 minute, the remaining solution was removed and the samples were air dried and examined at $80 \mathrm{KV}$ [14].

\section{Particle size, polydispersity index and zeta-potential of niosomes}

Nanoparticle size distribution, polydispersity index and zetapotential were determined using photon correlation spectroscopy (Zeta Potential Analyzer; Malvern Zetasize Nano-zs90, Malvern Instruments, Malvern, UK). The size distribution analysis was performed at a scattering angle of 90 degrees and at a temperature of $25^{\circ} \mathrm{C}$ using samples appropriately diluted with dispersant water. For each sample, the mean diameter \pm standard deviation of 10 determinations was calculated applying multimodal analysis [15]

\section{Entrapment efficiency (EE \%) of SS in niosomes}

Two mg of SS niosomes was mixed with $10 \mathrm{ml}$ of absolute alcohol; $0.1 \mathrm{ml}$ of the resultant SS niosomal dispersion was diluted with absolute alcohol and sonicated for ten minutes to obtain a clear solution. The concentration of entrapped SS was determined spectrophotometrically at $276 \mathrm{~nm}$ using UV spectrophotometer against the sample withdrawn from empty niosomal dispersion treated in a similar manner. The entrapment efficiency was determined relative to the original drug concentration as $(\mathrm{EE} \%=$ $\mathrm{ED} / \mathrm{TD} * 100$, Equation 1) Where EE \% is the entrapment efficiency percent, ED is the entrapped drug concentration and TD is the theoretical drug concentration [16].

\section{In vitro release of SS from niosomes}

The release of SS from niosomes was determined using the membrane diffusion technique [17-18]. One ml phosphate buffer $(\mathrm{pH} 7.4)$ was used to suspend SS; equivalent to $10 \mathrm{mg}$. The suspension was transferred to a glass tube with a soaked cellulose membrane that enclosed its lower end. Then, the glass tube was placed in a beaker containing $50 \mathrm{ml}$ phosphate buffer $(\mathrm{pH}$ 7.4) that was maintained at a temperature of $37^{\circ} \mathrm{C}$ [19]. Using shaker water bath; the beaker was kept under mild agitation ( $50 \mathrm{rpm}$ ). Aliquots were withdrawn at predetermined time intervals for $8 \mathrm{~h}$, then the drug concentration was determined at $276 \mathrm{~nm}$. The experiment was run in triplicates. The obtained data were kinetically analyzed to determine the pattern of the drug release. For better characterization of the drug release behaviour for the systems studied and to understand the corresponding mechanism, Korsmeyer-Peppas semi-empirical model was applied [20-21].

\section{Preparation of MDI}

Two formulae were used. MDI (F1), containing $20 \mathrm{mg}$ SS $(0.01 \%$ of the whole canister) in propellant HFA and considered the control batch, while MDI (F2) containing the REV SS niosomes (table 1). The prescribed quantity of the selected niosomes (20 mg SS) was suspended in the propellant HFA that was used as a solvent applying soya leicithin as a dispersing agent and then quantitatively placed in the aerosol container, the valve assembly was inserted and crimped into place, and pressure was introduced into the container equal to propellant vapor pressure from a pressure burette. When the pressure in the container equals that in the burette, the process was stopped. The desired pressure was obtained by increasing the pressure in the filling apparatus through the use of compressed air, using the typical pamasol packaging line that was used to fill the metered dose aerosol inhalers.

Table 1: Composition of SS alone and SS niosomes for aerosol studies

\begin{tabular}{llllll}
\hline Formula & \multicolumn{2}{l}{ Amount of ingredients in $\mathbf{( g )}$} & Molar ratio & \multicolumn{2}{l}{ Amount of SS in the whole canister } \\
\cline { 2 - 6 } & SS & Span60: cholesterol & Span60: cholesterol & (mg) & (\%) \\
\hline F1 & 0.02 & - & - & 20 & 0.01 \\
F2 & 1.5 & $6.4: 5.6$ & $1: 1$ & 20 & 0.01 \\
\hline
\end{tabular}

Note: Niosomes in formula 2 (F2) are entrapping 20 mg SS, (SS): Salbutamol Sulphate

\section{Characterization of niosomal MDI according to US pharma- copeia, 2004}

The particle size of niosomal aerosol was measured by laser diffraction Malvern Mastersizer as a suitable alternative to impaction methods [22]. Particle size analysis of each sample was performed with reference particle refractive index $(1.5295,0.1000)$ and reference dispersant refractive index (1.3300). The particle size of niosomal aerosol is converted into a volume based size according to a reported method [23]. The residual volume was $1.820 \%$. The preparation was appropriately diluted with purified water inside the Malvern sample dispersion unit for analysis using the $300 \mathrm{RF}$ lens as recommended by the manufacturer, and laser radiation unit class $3 \mathrm{~B}$ with a measurement beam lens of $2.40 \mathrm{~mm}$ and a range lens of $45 \mathrm{~mm}$ for each measurement attached to a measuring cell. The obscuration level was kept between $40-55 \%$ at a stable count rate. The average particle size was determined from triplicate of each sample.

Leakage rate was carried out using ten aerosol containers. Each container was weighed $\left(\mathrm{W}_{1}\right)$ then allowed to stand in an upright position at a temperature of $25.0 \pm 2.0^{\circ} \mathrm{C}$ for $3 \mathrm{~d}$. Each container was weighed again $\left(\mathrm{W}_{2}\right)$ then; the $\mathrm{h}(\mathrm{T})$ during which the containers under test were recorded. The leakage rate $(\mathrm{mg} /$ year $)$ of each container was calculated by the formula of $365^{*}(24 / \mathrm{T}) *\left(\mathrm{~W}_{1}-\mathrm{W}_{2}\right)$.

To calculate weight per puff, the valve of each container was actuated for 10 puffs, and then; each container was weighed again (W2). The total weight loss from each canister was calculated (W1-W2). The total number of puffs was determined by counting the number of priming discharges until the container was empty using 10 cans.
Dose uniformity was determined using a reported method [24] in which a collection tube covered with a tubing connector and a mouthpiece adaptor was tested. Using a mouthpiece adaptor ensured that the tip of the inhaler mouthpiece was flushed with the open end of the sample collection tube. Each canister had been shaken well, four puffs were discharged away in the air then the minimum recommended dose was discharged into the apparatus through the mouthpiece adaptor by depressing the valve four times allowing at least 10 seconds between doses to ensure that the dose has been completely discharged. The inhaler was detached from the apparatus; the content of the apparatus was assayed for drug spectrophotometrically at $276 \mathrm{~nm}$ after rinsing the interior of the apparatus with $10 \mathrm{ml}$ methanol against the blank sample.

The drug content over the entire can in each of 10 separate containers was also determined. The stem valve and mouthpiece were removed with a special device then the whole canister content was diluted with methanol; dose uniformity over the entire content was assayed spectrophotometrically at $276 \mathrm{~nm}$ against the blank sample.

\section{RESULTS AND DISCUSSION}

\section{Characterization of niosomal vesicles}

\section{Entrapment efficiency (EE \%)}

The EE\% of SS in niosomes was found to be $66.19 \% \pm 0.48$. This result was in accordance with [25] who reported that REV method is the most efficient method used to entrap water-soluble drug. Furthermore, [26] reported that REV method used to prepare Hydroxychloroquine in niosomes achieved the highest entrapment efficiency (86.4\%). Reverse- 
phase evaporation method has these unique advantages for encapsulating water-soluble materials such SS as the organic solvent is simply removed from the inverted micelles resulting in vesicles with larger aqueous space to lipid ratio and consequently higher $\mathrm{EE} \%$.

\section{Particle size, polydispersity index and zeta-potantial of niosomes}

A particle size ranged from 400-450 nm (fig. 1. a) with a dispersant RI 1.330 and viscosity (cP) equals 0.8872 was considered optimum for pulmonary application. Polydispirsity index (Pdi) value was 0.354 that indicates the homogenous distribution of formed niosomes (fig. 1. a). Zetapotential of RE niosomes was-46.5 which reflects the stability of formed niosomes (fig. 1. b), [27].

\section{Scanning electron microscopy (SEM)}

SEM illustrated surface characteristics of REV SS-loaded niosomes (fig. 1. c) which appeared spherical with some discontinuities in the membrane. This may be explained as the acyl-chain structure of span 60 could affect cholesterol interactions causing variations in cholesterol distribution. The polar head group of non-ionic surfactant must cover the non-polar portion of cholesterol; this coverage is essential to avoid the unfavourable free energy of cholesterol that when contacts with water decrease the repulsion between cholesterol molecules [28].

\section{Transmission electron microscopy (TEM)}

The morphology of the selected formula of REV SS-loaded niosomes (fig. 1. d), revealed the presence of well identified spherical multilamellar vesicles existing in disperse pattern.

Fig. 1: Particle size (a), zeta-potential (b), scanning electron microscopy (c) and transmission electron microscopy (d) of REV SS niosomes. (a)

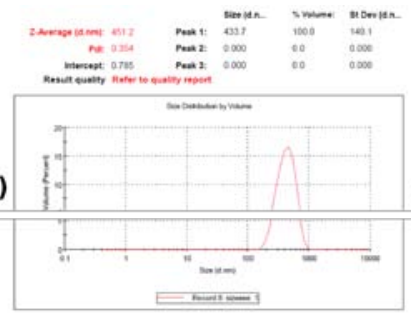

(c)

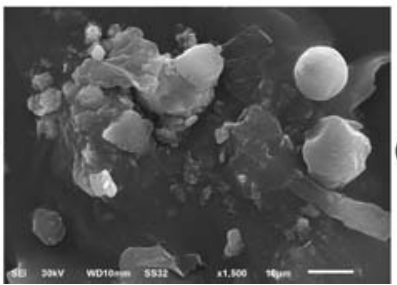

(b)

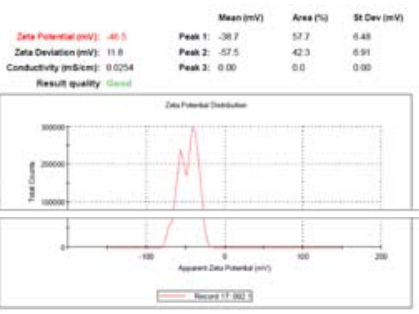

(d)

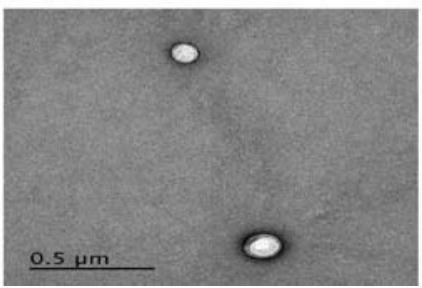

Fig. 1: The release profile of REV niosomal SS

The results illustrated in (fig. 2. a) showed that SS release percent after eight hours from REV SS niosomes was $76.54 \% \pm 0.132$. Furthermore, the slow release rate depends on both polymer breakdown and diffusion out of the matrix. Correlation of release data revealed that the release profile followed Higuchi model with regression parameters of $\mathrm{r}^{2}=0.992$, for REV
SS niosomes (fig. 2. b). This kinetic pattern indicated that SS release is dominated by diffusion model which normally depends on drug concentration gradient between nano-vesicles and dissolution media with penetration of this media through a porous wall which accompanied by matrix disruption [29].

(a)

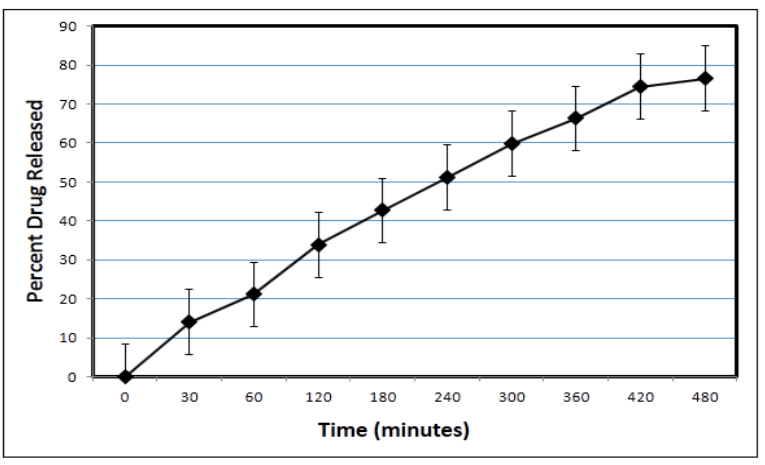

(b)

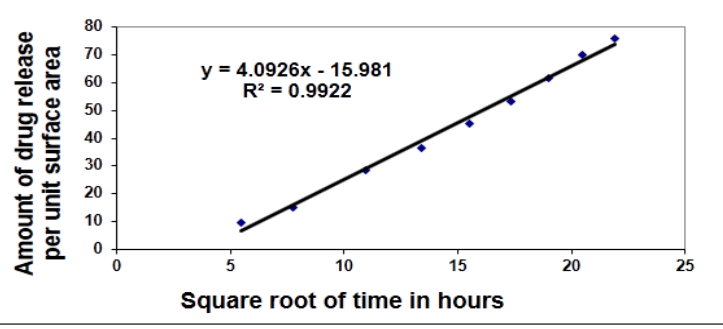

Fig. 2: (a) The release of REV SS from niosomes of span60 and cholesterol in the molar ratio 1:1 in phosphate buffer pH 7.4 at $37^{\circ} \mathrm{C}$. (b) Higuchi diffusion model for the release of $S S$ from niosomal vesicles, sqrt ( $t$ ): square root of time in hours, amount/S. A: amount of drug release per unit surface area at time $t$. $n=3$, data are expressed as mean $\pm S D$ 
In this light, release can be changed by varying system surface area and wettability; determined by size and uniformity. Meanwhile, loading percentage directly affects the drug concentration gradient and release rate [30]. Further kinetic studies were carried out and the release pattern was non fickian with $\mathrm{n}=0.6929$ for $\mathrm{N}$ (table 2).

Table 2: Release kinetics of SS from different niosomal formulations

\begin{tabular}{llllll}
\hline \multirow{2}{*}{ Formula } & \multicolumn{1}{l}{ Higuchi diffusion } & \multicolumn{3}{l}{ Korsmeyer-peppas } & Mechanism of drug release \\
\cline { 2 - 5 } & $\mathbf{R}^{\mathbf{2}}$ & $\mathbf{R}^{\mathbf{2}}$ & $\mathbf{K}$ & $\mathbf{n}$ & \\
\hline $\mathrm{N}$ & 0.992 & 0.999 & 0.006 & 0.6929 & Non Fickian \\
\hline
\end{tabular}

Note: formula $(\mathrm{N})$ : Niosomes containing salbutamol sulphate (SS) that prepared by reversed-phase evaporation method (REV), $\left(\mathrm{R}^{2}\right)$ : linear regression, (K): a constant incorporating characteristics of the particle system, (n): the diffusion exponent

\section{Characterization of niosomal MDI according to USP}

Characterization of niosomal MDI was performed. Statistical analysis using one way ANOVA $(\mathrm{p}<0.05)$ was carried out by graphpad prism6 ${ }^{\circledR}$ showing that the results complies with pharmacopeial specifications according to USP 2004. Fig. (3) Illustrates the particle size of aerosolized REV SS niosomes which ranged from 0.64 to 4.51 $\mu \mathrm{m}$. The results also indicate that niosomal SS particle size measured by (TEM) and (SEM) were in good agreement with malvern mastersizer results, which was almost below $5 \mu \mathrm{m}$. Particles larger than $5 \mu \mathrm{m}$ are rapidly removed from the lung by coughing or swallowing while particles smaller than $0.5 \mu \mathrm{m}$ may escape impaction in the upper airways, thus only aerosols $3-5 \mu \mathrm{m}$ in diameter show efficient penetration into the lungs [31]. All characterization parameters were studies according to US Pharmacopeia, 2004 [32].
The results illustrated in (table 3) showed that, weight varies from 22.65-23.69 gm with no significant differences between the 20 cans $(p<0.05)$ as $(23.42 \pm 0.24)$ for SS and $(20.65 \pm 0.33)$ for niosomal SS. Weight per puff (mg) of 10 canisters of SS and niosomal SS were found to be $(83 \pm 0.31)$ and $(80 \pm 0.27) \mathrm{mg}$, respectively which is not less than $71 \mathrm{mg}$ and not more than $83 \mathrm{mg} /$ puff. Leakage test (mg/year) showed $(425 \pm 1.89)$ for SS and $(472 \pm 0.56)$ for niosomal SS. Number of puffs per canister (delivery/canister) for 10 containers showed $(190 \pm 0.53)$ and $(215 \pm 0.61)$ (del/can) for SS and niosomal SS respectively. Regarding dose uniformity per puff, the results showed $(115 \% \pm 0.19)$ for SS and $(95 \% \pm 0.55)$ for niosomal SS, this in accordance with the recorded values in USP that ranged from $85 \%$ to $115 \%$ using 10 canisters. Moreover, the results of dose uniformity per the whole canister showed that $(99 \% \pm 0.45)$ and $(105 \% \pm 0.11)$ for SS and niosomal SS, respectively which meet the compendia requirements that ranged from $85 \%$ to $115 \%$ using 10 canisters.

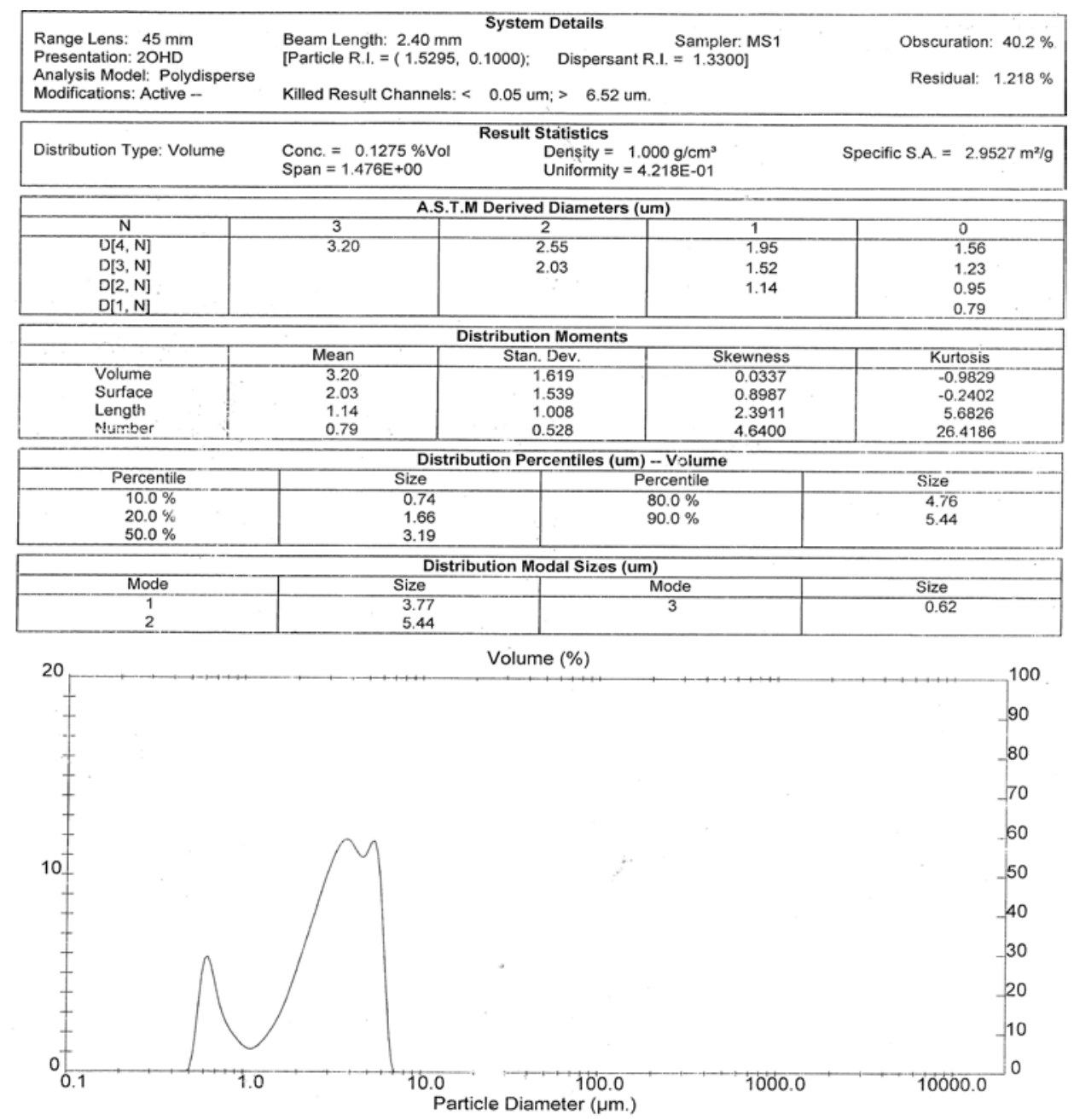

Fig. 3: Malvern mastersizer report of the particle size of REV SS niosomes in MDIs Note: $D(4, N), D(3, N), D(2, N), D(1, N)$ are the equivalent derived diameter at $90,80,50$ and $10 \%$ cumulative volume 
Table 3: Pharmacopeial specifications of the prepared aerosols

\begin{tabular}{llll}
\hline \multirow{2}{*}{ Test } & Salbutamol sulphate aerosol & Niosomal salbutamol sulphate aerosol & Pharmacopeial specifications \\
\cline { 2 - 4 } & Limit & $472 \pm 0.56$ & Comply \\
\hline Leakage test (mg/year) & $425 \pm 1.89$ & $20.65 \pm 0.33$ & Comply \\
Weight variations (g) & $23.42 \pm 0.24$ & $80 \pm 0.27$ & Comply \\
Weight per puff (mg) & $83 \pm 0.31$ & $215 \pm 0.61$ & Comply \\
Number of puffs/can (del/can) & $190 \pm 0.53$ & $95 \pm 0.55$ & Comply \\
Dose uniformity/puff\% & $115 \pm 0.19$ & $105 \pm 0.11$ & Comply \\
Dose uniformity/can\% & $99 \pm 0.45$ & & \\
\hline
\end{tabular}

Note: All values were represented as mean $\pm \mathrm{SD}(\mathrm{n}=3), P<0.05$

\section{CONCLUSION}

The findings of this investigation showed that; niosomes prepared by REV method entrapped high amount of water soluble SS. REV SS niosomes can be formulated as MDI with physical properties met the USP requirements, and so it could be delivered to lung as targeting delivery systems, which maximize the delivery of the drug while minimize its clearance, that guarantees controlled drug delivery with reduced number of dosing which helps to reduce the side effects. It also offers a great opportunity for deposition and retention of water-soluble compounds in the lung with great potential to relieve asthma symptoms. In this light, our future studies will use the current results in translating this technology in clinics after conducting further in vivo investigations.

\section{Research involving human and animal participants}

This article does not contain any studies with human and animal subjects performed by any of the authors.

\section{AUTHORS' CONTRIBUTION}

Dr Mona Arafa conceived and designed the study, conducted the formulation and characterization of SS-loaded niosomes and the two proposed MDIs, collected and analyzed the data in addition to statistical analysis. Both Dr Mona Arafa and Dr. Bassam Ayoub reviewed the literature, performed the spectrophotometric analysis. Finally, all authors wrote, reviewed and approved the manuscript including fig. and tables.

\section{ACKNOWLEDGMENT}

The authors are grateful for Dr. Abd-Elsabour Ahmedhead of the Aerosol Department, Arab drug company, Cairo 11813, Egypt for the MDIs packaging.

\section{CONFLICT OF INTERESTS}

The authors declare no competing interests. We declare that this work was done by the authors named in this article and all liabilities pertaining to claims relating to the content of this article will be borne by the authors.

\section{REFERENCES}

1. Patton JS, Bukar J, Nagarajan S. Inhaled insulin. Adv Drug Delivery Rev 1999;35:235-47.

2. Kaparissides C, Alexandridou S, Kotti K, Chaitidou S. Recent advances in novel drug delivery systems. J Nanotechnol 2006;2:1-11.

3. Wall DA. Pulmonary absorption of peptides and proteins. Drug Delivery 1995;2:1-20.

4. Anselmo AC, Mitragotri S. An overview of the clinical and commercial impact of drug delivery systems. J Controlled Release 2014;190:15-28.

5. Taburet AM, Schmit B. Pharmacokinetic optimization of asthma treatment. Clin Pharmacokinetics 1994;26:396-418.

6. El-najjar BY, Hussain SA. Chitosan microspheres for the delivery of chemotherapeutic agents: paclitaxel as a model. Asian J Pharm Clin Res 2017;10:15-9.

7. Marianecci C, Di Marzio L, Rinaldi F, Celia C, Paolino D, Alhaique F, et al. Niosomes from 80s to present: the state of the art. Adv Colloid Interface Sci 2014;205:187-206.

8. Duarah S, Pujari K, Durai RD, Narayanan VHB. Nanotechnologybased cosmeceuticals: a review. Int J Appl Pharm 2016;8:8-12.
9. Morgan D, Paull J, Richmond B, Wilson-Evered E, Ziccone S. Pharmacokinetics of intravenous and oral salbutamol and its sulphate conjugate. Br J Clin Pharmacol 1986;22:587-93.

10. Moxham J, Costello JF. Textbook of medicine. ed. Souhami RL, Moxham J. 506 (Chapter 15), (3rd ed., Churchill Livingstone, London, 1997); 1997.

11. Hutchings $M$, Paull J, Wilson-Evered E, Morgan D. Pharmacokinetics and metabolism of salbutamol in premature labour. Br J Clin Pharmacol 1987;24:69-75.

12. Hua W, Liu T. Preparation and properties of highly stable innocuous niosome in Span 80/PEG 400/H2O system. Colloids Surf A 2007;302:377-82.

13. Szoka JF, Papahadjopoulos D. Procedure for preparation of liposomes with large internal aqueous space and high capture by reverse-phase evaporation. Proc Natl Acad Sci USA 1987;75:4194-8.

14. Abd-Elbary A, El-laithy HM, Tadros MI. Sucrose stearate-based proniosome-derived niosomes for the nebulisable delivery of cromolyn sodium. Int J Pharm 2008;357:189-98.

15. Fonte P, Soares S, Sousa F, Costa A, Seabra V, Reis S, et al. Stability study perspective of the effect of freeze-drying using cryoprotectants on the structure of insulin-loaded into PLGA nanoparticles. Biomacromolecules 2014;15:3753-65.

16. Arafa MG, Ayoub BM. DOE optimization of the nano-based carrier of pregabalin as hydrogel: new therapeutic and chemometric approach for controlled drug delivery systems. Sci Rep 2017;7:41503.

17. Devaraj GN, Parakh SR, Devraj R, Apte SS, Rao BR, Rambhau D. Release studies on niosomes containing fatty alcohols as bilayer stabilizers instead of cholesterol. J Colloid Interface Sci 2002;251:360-5.

18. Glavas-Dodov M, Goracinova K, Mladenovska K, FredroKumbaradzi E. Release profile of lidocaine $\mathrm{HCl}$ from topical liposomal gel formulation. Int J Pharm 2002;242:381-4.

19. Gursoym A, Kut E, Ozkirmli S. Co-encapsulation of isoniazid and rifampicin in liposomes and characterization of the liposome by derivative spectroscopy. Int J Pharm 2004;271:115-23.

20. Korsmeyer RW, Gurny R, Doelker E, Buri P, Peppas NA. Mechanisms of solute release from porous hydrophilic polymers. Int J Pharm 1983;15:25-35.

21. Kulkarni A, Mandhare T, Aloorkar N. Application of novel natural polymer for controlling the release of fenoverine from controlled release matrix tablets. Int J Appl Pharm 2017;9:1-9.

22. De-Boer AH, Le Brun PPH, Van Der Woude HG, Hagedoorn P, Heijerman HGM, Frijlink HW. Dry powder inhalation of antibiotics in cystic fibrosis therapy, part 1: development of a powder formulation with colistin sulfate for a special test inhaler with an air classifier as a de-agglomeration principle. Eur J Pharm Biopharm 2002;54:17-24.

23. Jones SA, Martin GP, Brown MB. High-pressure aerosol suspensions-a novel laser diffraction particle sizing system for hydrofluroalkane pressurized metered dose inhalers. Int J Pharm 2005;302:154-65.

24. Marie PF, Pierre L, Anne G. The influence of carrier roughness on adhesion, content uniformity and the in vitro deposition of terbutaline sulphate from dry powder inhalers. Int J Pharm 2004;275:201-9.

25. Moghassemi S, Hadjizadeh A. Nano-niosomes as nanoscale drug delivery systems: an illustrated review. J Controlled Release 2014;185:22-36 
26. Bendas ER, Abdullah $\mathrm{H}$, El-Komy MHM, Kassem MAA. Hydroxychloroquine niosomes: a new trend in topical management of oral lichen planus. Int J Pharm 2013;458:287-95.

27. Jassem NA, Rajab NA. Fomulation and invitro evaluation of azilsartan medoxomil nanosuspension. Int J Pharm Pharm Sci 2017;9:110-9.

28. Parker A, Miles K, Cheng K, Huang J. Lateral distribution of cholesterol in dioleoylphosphatidylcholine lipid bilayers: cholesterol-phospholipid interactions at high cholesterol limit. Biophys J 2004;86:1532-44.
29. Davies NM, Feddah MR. A novel method for assessing dissolution of aerosol inhaler products. Int $\mathrm{J}$ Pharm 2003;255:175-87.

30. Hanif SN, Garcia-Contreras L. Pharmaceutical aerosols for the treatment and prevention of tuberculosis. Front Cell Infect Microbial 2012;2:118-24

31. Glyn T, Ian K. Pulmonary drug delivery, chapter 10 in drug delivery and targeting. Anya $\mathrm{MH}$, Andrew WL, James S. ed. Taylor and Francis, Inc, New York; 2001.

32. US Pharmacopeial Convention 27, Rockville, MD; 2004. p. 2253-8. 